\title{
Article \\ Blood Culture Contamination: A Single General Hospital Experience of 2-Year Retrospective Study
}

\author{
Anna Tenderenda ${ }^{1, *(\mathbb{D})}$, Monika Lysakowska ${ }^{2}$, Robert Dargiewicz ${ }^{3} \mathbb{1}$ and Anna Gawron-Skarbek ${ }^{1, *(D)}$ \\ 1 Department of Geriatrics, Medical University of Lodz, 90-647 Lodz, Poland \\ 2 Department of Microbiology and Medical Laboratory Immunology, Medical University of Lodz, \\ 90-213 Lodz, Poland; monika.lysakowska@umed.lodz.p1 \\ 3 Department of Physiotherapy, Academy of Medical Science in Bialystok, 15-875 Bialystok, Poland; e@kiero.net \\ * Correspondence: anna.tenderenda@stud.umed.lodz.pl (A.T.); anna.gawron@umed.lodz.pl (A.G.-S.)
}

Citation: Tenderenda, A.; Łysakowska, M.; Dargiewicz, R.; Gawron-Skarbek, A. Blood Culture Contamination: A Single General Hospital Experience of 2-Year Retrospective Study. Int. J. Environ. Res. Public Health 2022, 19, 3009. https://doi.org/10.3390/ ijerph19053009

Academic Editors: Izabella Lecka, Sally Brailsford, Józef Haczyński and Remigiusz Kozlowski

Received: 30 December 2021 Accepted: 28 February 2022 Published: 4 March 2022

Publisher's Note: MDPI stays neutral with regard to jurisdictional claims in published maps and institutional affiliations.

Copyright: (C) 2022 by the authors. Licensee MDPI, Basel, Switzerland. This article is an open access article distributed under the terms and conditions of the Creative Commons Attribution (CC BY) license (https:// creativecommons.org/licenses/by/ $4.0 /)$.

\begin{abstract}
In the event of blood culture contamination (BCC), blood culture (BC) needs to be repeated This may delay appropriate treatment, prolong hospitalization and, consequently, increase its costs. The aim of the study was to assess the frequency of BCC and associated factors in a general hospital in Poland based on reports of BC in samples submitted for laboratory testing in 2019-2020. BCC is recognized when bacteria (especially those belonging to natural human microbiota) are isolated from a single sample and no clinical signs indicated infection. True positive BC is confirmed by the growth of bacteria in more than one set of blood samples with the corresponding clinical signs present. The structure of BC sets, microorganisms, and laboratory costs of BCC were analyzed. Out of 2274 total $\mathrm{BC}$ cases, $11.5 \%$ were true positive $\mathrm{BC}$ and $9.5 \%$ were $\mathrm{BCC}$. Of all the BCC identified in the entire hospital, $72 \%$ was from Internal Medicine (IM) and Intensive Care Unit (ICU) combined. When single sets for BC were used in IM in 2020, the use increased to 85\% compared with $2019(p<0.05)$. The predominant isolates were coagulase-negative staphylococci $(84 \%)$. The estimated extra laboratory costs of BCC exceeded EUR 268,000. The BCC was a more serious problem than expected, including non-recommended using of single $\mathrm{BC}$ sets. Compliance with the $\mathrm{BC}$ collection procedure should be increased in order to reduce BCC and thus extra hospital costs.
\end{abstract}

Keywords: blood culture contamination; bloodstream infections; coagulase-negative staphylococci

\section{Introduction}

One of the key tools available to clinicians for differentiating clinical presentations of bloodstream infections (BSIs) in patients is microbiological diagnosis. Blood culture (BC) can be used to confirm infectious etiology, isolate etiologic agents, and determine their drug susceptibility, and can form the basis for the implementation of targeted therapy [1-3].

The use of less effective empirical therapy, including broad-spectrum antibiotics, places greater financial burdens on the healthcare provider and can increase drug resistance [4]. Furthermore, targeting the specific pathogen and employing focused therapy can significantly improve the final outcome [5]. Delayed appropriate treatment may result in prolonged hospitalization, inappropriate antibiotic therapy, and consequently greater exposure to drug side effects and toxicity [4-6].

In clinical practice, false positive cultures are sometimes reported following contamination with human microbiota; these are known as blood culture contaminations (BCCs). However, distinguishing true BSI from contamination remains a challenge for physicians and microbiologists $[7,8]$. The contamination rate can be calculated in two methods. The blood culture contamination rate (\%) was calculated according to the following formula: (1) the number of BCC $\times 100 \%$ to the number of total positive BC (true and false positive $B C)$, (2) the number of BCC $\times 100 \%$ to the number of total BC (true positive BC, false positive $\mathrm{BC}$, true negative $\mathrm{BC}$, false negative $\mathrm{BC}$ ). Despite the development of culture 
techniques and automation of the identification process, some laboratories report BCC rates of nearly $50 \%$ of total positive BC $[7,8]$. Clinical and Laboratory Standards Institute recommendations indicate that contamination rates for adults in health care settings should not exceed $3 \%$, measured as the total number of blood cultures (total BC) $[9,10]$. Some authors record $\mathrm{BCC}$ rates ranging from $0.6 \%$ to $6 \%$, which only emphasizes the magnitude of the problem $[11,12]$. False positive BC events increase the cost of additional laboratory tests, by up to $20 \%$, cause unwarranted antibiotic prescription, and are associated with a $40 \%$ increase in antimicrobial spending. BCC also results in prolonged hospitalization, i.e., of up to 5 days [9-13]. Studies in the United States estimate the hospital costs associated with BCC to range from USD 2844 to USD 10,078 [8,13].

In order to effectively prevent the consequences of $\mathrm{BCC}$, it is first necessary to understand and eliminate its causes. In addition, for individual health care units, there is a need to identify the key critical issues.

Standard precautions must be taken during the sampling process, aseptic conditions must be rigorously maintained, and all rules included in the procedures must be strictly followed. One of the most important causes of BCC is the use of improper techniques for collecting material for laboratory testing, e.g., incorrect use of protective gloves, improper preparation of media bottles, or taking samples in an order that does not conform to the protocol [13-17].

It is equally important that the injection site is correctly disinfected when taking samples, e.g., the preparation must be left on the skin long enough and the disinfected site should not be touched. Failure to follow these basic rules results in the transfer of bacteria physiologically present on the skin to the culture medium bottles and a subsequent false positive result $[16,17]$.

The results of a number of clinical laboratory investigations indicated that certain microorganisms are more likely to contaminate blood samples. These include coagulasenegative staphylococci (CoNS), including Staphylococcus epidermidis, most Corynebacterium species, Bacillus spp., Propionibacterium spp., Micrococcus spp., and Cutibacterium acnes $[9,16,18,19]$. These microorganisms are commonly found on the skin, and although they can cause serious infections under certain conditions, their detection in a single BC is considered a contaminant without clinical significance [16,20].

To help differentiate between contamination and true bacteremia, a sufficient number of BC diagnostic sets should be collected; a set is defined as two bottles of culture medium: one aerobic and one anaerobic. It is also generally recommended that two- or three-bottle sets are used; the sensitivity of a single blood culture set is limited due to the periodic appearance of bacteria and fungi in the bloodstream [21]. A study by Lee et al. found that BC sensitivity increased when more than one blood set was used; in addition, contamination usually occurs in a single bottle from a set, while cases of BSI demonstrate positivity in several samples [22]. Another reason for taking cultures in multiple sets, from anatomically different sites, is that while contamination usually occurs in a single bottle from a set, cases of BSI demonstrate positivity in several samples [22,23].

There is a large gap in the current knowledge regarding the problem with BCC in hospitals in Poland. As such, the aim of the study was to evaluate its incidence in a general hospital. The study itself included the following critical data for clinically insignificant results: time of sampling (month, day of the week, a shift/day-night), a quantitativequalitative analysis of the microorganisms isolated from BCC, the choice of the number of diagnostic sets used, and the costs resulting from the contamination.

\section{Materials and Methods}

\subsection{Study Design and Data Collection}

The study consisted of a retrospective analysis of BC results collected by a microbiology laboratory between 1 January 2019 to 31 December 2020. The study was conducted in a general hospital (16 departments; 380 beds) (Subcarpathian Voivodeship, Tarnobrzeg, Poland). The data collection process, including the conduct of microbiological tests, criteria 
for diagnosing BSI and BCC and the report-generation procedure, were standardized according to the laboratory procedures.

A total of 2274 blood cultures were analyzed. True positive BC was defined as a case in which a microorganism potentially representing an etiological agent of BSI was cultured, consistent with the patient's clinical condition. A laboratory result found to be true positive was labeled with the alert microorganism. To assess the clinical significance of organisms that are often considered contaminants when isolated from blood cultures (coagulasenegative staphylococci, Corynebacterium spp., Bacillus spp., and Propionibacterium spp.), the clinical microbiology laboratory used an algorithm [21]. If there is a suspicion of BCC, an additional sample should be collected within $48 \mathrm{~h}$, after a microbiologist reports the first sample as probably contaminated. When no additional blood cultures are obtained from a patient within $48 \mathrm{~h}$, a microbiologist discusses the potential significance of the isolate with a physician caring for the patient. On the basis of the assessment clinical history, disease symptoms, and additional laboratory tests, the isolate was classified as a pathogen or as a contaminant.

As a number of single BC sets were sent for microbiological analysis, despite recommendations, any single positive blood sample in which natural microbiota isolated was identified for consultation with the main doctor. If no clinical markers reflecting infection were present, the culture was considered as BCC. As only single BC sets were investigated in many cases, this contact with the main doctor was crucial to establish whether the other results confirm the infection. The absence of pathogen growth in the collected specimen indicated a negative culture [24].

\subsection{Methods}

\subsubsection{Blood Culture Technique}

Blood for culture was collected on liquid media in BACTEC bottles: AERO (aerobic culture), ANAERO (anaerobic conditions), or PEDS (pediatric, dedicated for children), and then incubated in a BD BACTEC 9240/9120 (Becton, Dickinson and Company, Franklin Lakes, NJ, USA, 2012) for 5 days. The instrument detects any increase in the level of carbon dioxide produced by the microorganisms, typically identifying a positive sample during the first 2 or 3 days of incubation. In the next step, a gram-stained direct preparation was made to provide initial information about the microorganisms. The culture from positive blood cultures was streaked onto appropriate solid media. From the BACTEC AERO bottle, the specimen was inoculated onto COS (Columbia agar with Sheep Blood) and CPS (Chromagar Orientation) (BioMérieux, Marcy-l'Étoile, France) media and incubated at $35 \pm 2{ }^{\circ} \mathrm{C}$ for $18-24 \mathrm{~h}$ under aerobic conditions. From a BACTEC ANAERO bottle, blood samples were cultured on the SCS (Schaedler Agar $+5 \%$ Sheep Blood) and SNVS (Schaedler Neo. Vanco. Agar $+5 \%$ Sheep Blood) (BioMérieux) medium; these were incubated under anaerobic conditions at $35 \pm 2{ }^{\circ} \mathrm{C}$ for $18-24 \mathrm{~h}$. The cultures on COS and CPS media were also performed under the same conditions as for aerobic bottles. At the end of the incubation, the morphology (colony structure) and type of culture (aerobic, anaerobic) were determined, the cultured microorganisms were identified, and their drug susceptibility evaluated according to 2019 and 2020 EUCAST recommendations with the Vitek system (BioMérieux) [25].

\subsubsection{Microbiological Reports}

The results of blood tests and annual statements with details of hospital departments were obtained using a laboratory information system (LIS Centrum MARCEL S.A., Zielonka, Poland). The reports provided data on, inter alia, the number of Total $\mathrm{BC}$, the number of $B C C$, the number of true positive $B C$, the number of negative $B C$, the number of blood culture set, the date and time of specimen collection for testing.

Based on these findings, the following variables were selected: month, day of the week, and work shift (day-night); in the case of the latter, day shift was assumed to indicate on-call duty from 7:00 a.m. to 7:00 p.m. and night shift on-call duty from 7:00 p.m. to 
7:00 a.m. The week was divided into working days (Monday-Friday) and weekends (Saturday-Sunday). The laboratory results allowed for a qualitative and quantitative summary of the isolated microorganisms.

In further parts of the analysis, relating to the critical points of BCC occurrence, the frequency of $\mathrm{BCC}$ was presented as the rate of $\mathrm{BCC}$ with respect to all positive $\mathrm{BC}$ samples (true and false) according to international standards [10].

This study was reviewed and approved by the laboratory manager.

\subsubsection{Analysis of Laboratory Costs of Contamination}

The costs incurred by contamination were determined by calculating the additional materials needed to repeat the microbiological testing of $\mathrm{BC}$ by the laboratory. The analysis was performed using Excel (Microsoft Office 2019, Microsoft Corporation, Redmond, WA, USA). The cost simulation was performed for all hospital departments that had BCC in their microbiology reports. The calculation took into account the number of BCC generated by a given department, and the costs of used BC sets and materials (i.e., media, reagents, and antibiotics needed to perform an antibiogram). The analysis assumed that retesting is performed according to standard procedures, that is, using at least two sets of transport media (except for testing in neonates and children with body mass $<36.3 \mathrm{~kg}$ ) [24]. It was not possible to estimate the costs of treating patients in the departments: no permission was provided to collect the relevant data.

\subsection{Statistical Analysis}

The statistical analysis was performed using Statistica version 13.1 CSS software (StatSoft Polska Sp. z o.o., Kraków, Poland). A One-way analysis of variance (ANOVA) with the post hoc Fisher's LSD test was applied to classify departments by mean monthly BCC (BCC m-index), a Friedman ANOVA test was applied to evaluate the profiles of the blood culture collection sets used in the classified departments. BCC m-index was calculated using the formula BCC m-index $=\mathrm{N}_{\mathrm{BCC}}$ per month $/ \mathrm{N}_{\text {Total } \mathrm{BC}}$, where $\mathrm{N}=$ the number of BCC in the month, and $\mathrm{N}_{\text {Total } \mathrm{BC}}=$ the number of all blood cultures in over 2 investigated years. The Wilcoxon matched pairs test was used to compare the rate of BCC across months and days of the week, and the Chi-square test on day and night work shifts, and on working days (Monday-Friday) and the weekend (Saturday-Sunday). The level for statistical significance was set at $p<0.05$.

\section{Results}

\subsection{Characteristics of Blood Culture and Contamination throughout the Hospital}

Of all the BC samples received (2274) in the microbiology laboratory during the study period (2019-2020), 479 (21.06\%) were positive BC samples, including both real BC, i.e., true positive; and BCC, i.e., false positive. The data indicate that $217(9.54 \%$ of Total BC) samples appeared contaminated (8.81\% in 2019 and $10.62 \%$ in 2020$)$, while 262 (11.52\%) had actual bacteremia (being true positives), as shown in Table 1.

The majority of sets retrieved for testing were single sets $(88.08 \%)$ in both $2019(87.19 \%)$ and $2020(89.38 \%)$ (Table 1).

Table 1. General characteristics of blood cultures performed at the hospital in 2019-2020.

\begin{tabular}{|c|c|c|c|c|}
\hline \multicolumn{2}{|c|}{ Variable } & 2019 & 2020 & Total \\
\hline \multicolumn{2}{|c|}{ Total BC } & 1351 & 923 & 2274 \\
\hline \multicolumn{2}{|c|}{ True positive $\mathrm{BC}, \mathrm{n}(\%)$} & $162(11.99)$ & $100(10.83)$ & $262(11.52)$ \\
\hline \multicolumn{2}{|c|}{ True negative $\mathrm{BC}, \mathrm{n}(\%)$} & $1071(79.27)$ & $725(78.55)$ & $1796(78.98)$ \\
\hline \multicolumn{2}{|c|}{$\mathrm{BCC}, \mathrm{n}(\%)$} & $119(8.81)$ & $98(10.62)$ & $217(9.54)$ \\
\hline \multicolumn{2}{|c|}{ Rate of BCC (\%) } & 42.35 & 49.49 & 45.30 \\
\hline \multirow{2}{*}{ Sets retrieved, $\mathrm{n}(\%)$} & 1 & $1178(87.19)$ & $825(89.38)$ & $2003(88.08)$ \\
\hline & $\geq 2$ & $173(12.81)$ & $98(10.62)$ & $271(11.92)$ \\
\hline
\end{tabular}

$\mathrm{BC}-\mathrm{Blood}$ Culture, BCC-Blood Culture Contamination, Rate of BCC-number of BCC to true positive BC 


\subsubsection{Percentage of BCC in Hospital}

During the 2-year follow-up period, the departments, Internal Medicine (IM), Intensive Care Unit (ICU), Oncology, Infant Care, and Neurology, collected the highest number of BC samples compared with other departments (>130 samples), while also having a noticeably high number of BCC (>10). The IM and ICU departments together accounted for $71.89 \%$ of BCC in the entire hospital (IM: 52.07\%; ICU: 19.82\%) (Figure 1).

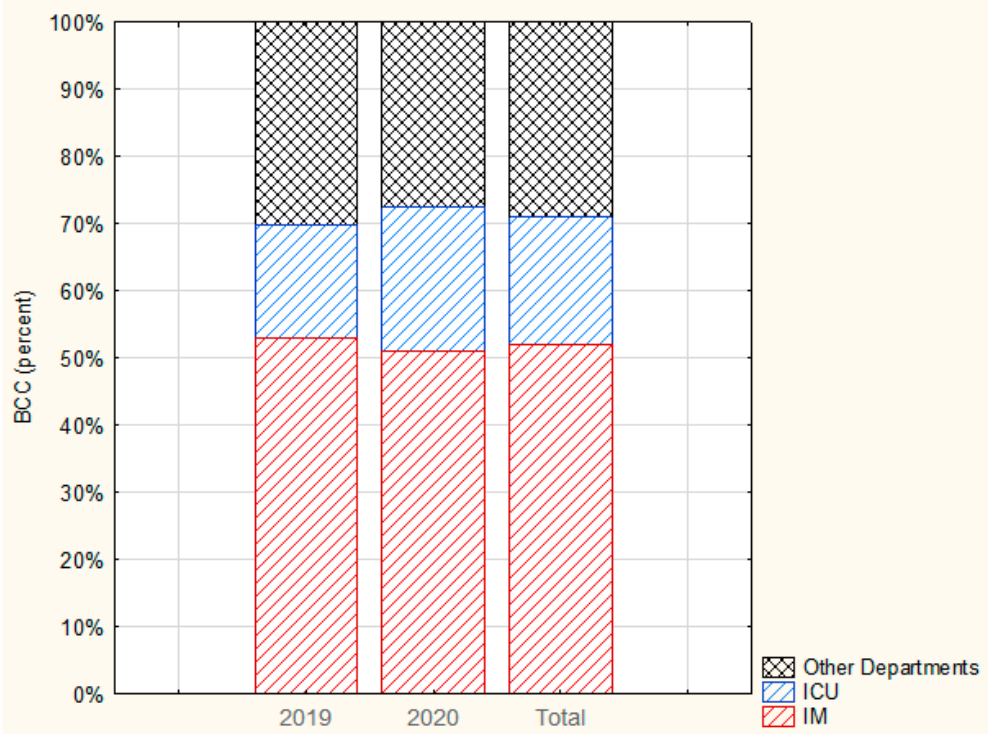

Figure 1. Percentage of BCC in IM, ICU and Other Departments in 2019 and 2020.

\subsubsection{Mean Monthly BCC Index in Hospital}

The IM and ICU departments demonstrated a significantly higher level of sample contamination compared with other departments. $(p<0.02)$, indicted by the distribution of BCC samples in relation to all tests performed in over 2 years. As no statistically significant differences were observed between the other departments in this regard $(p>0.05)$, they were not presented in detail (Figure 2).

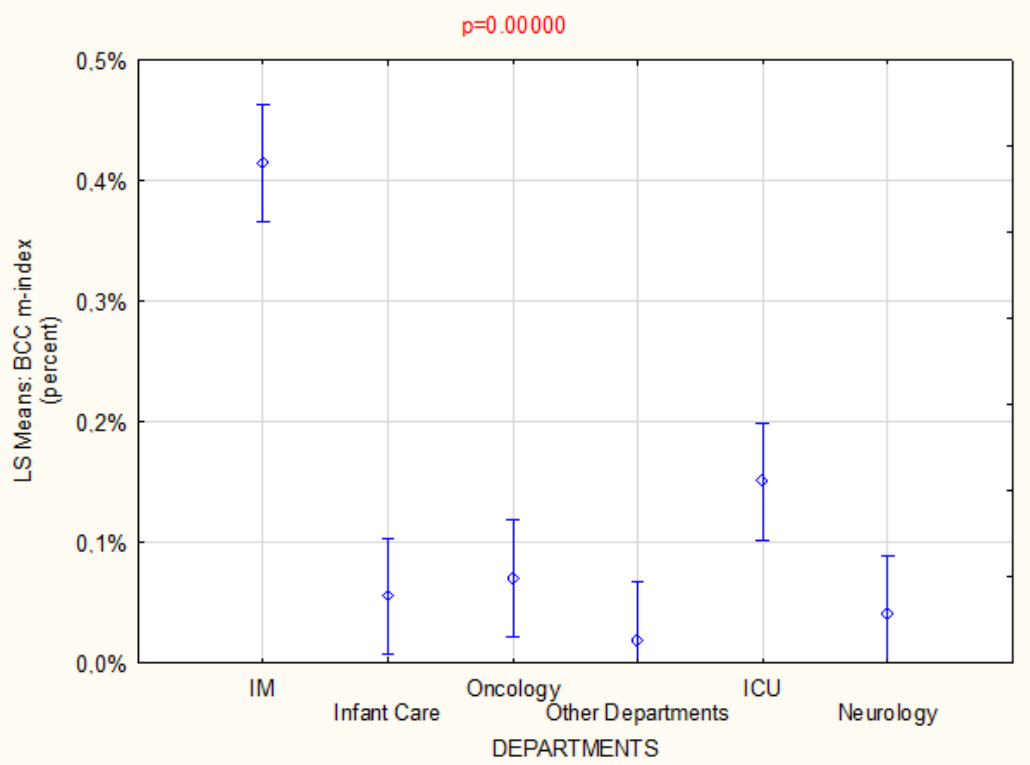

Figure 2. Characteristics of BCC m-index in hospital departments for the period of 2019-2020. 


\subsubsection{Number of BCC and Total BC Samples in Hospital}

Among the analyzed departments, some had no contamination (Pediatrics, Orthopedics, Infectious Disease, Surgery); however, these departments collected relatively few culture samples (Table 2).

Table 2. Number of BCC versus total BC samples among various hospital departments in 2019 and 2020.

\begin{tabular}{ccccccc}
\hline \multirow{2}{*}{ Department } & \multicolumn{3}{c}{ BCC } & \multicolumn{3}{c}{ Total BC } \\
\cline { 2 - 7 } & $\mathbf{2 0 1 9}$ & $\mathbf{2 0 2 0}$ & Total & $\mathbf{2 0 1 9}$ & $\mathbf{2 0 2 0}$ & Total \\
\hline Internal Medicine & 63 & 50 & 113 & 666 & 471 & 1137 \\
\hline ICU & 20 & 21 & 41 & 69 & 77 & 146 \\
\hline Oncology & 13 & 6 & 19 & 81 & 55 & 136 \\
\hline Infant Care & 9 & 6 & 15 & 209 & 138 & 347 \\
\hline Neurology & 2 & 9 & 11 & 79 & 66 & 145 \\
\hline Cardiology & 4 & 2 & 6 & 58 & 26 & 84 \\
\hline Infectious Disease & 6 & 1 & 7 & 33 & 17 & 50 \\
\hline Others & 2 & 3 & 5 & 156 & 73 & 229 \\
\hline
\end{tabular}

BC—Blood Culture, BCC—Blood Culture Contamination, ICU—Intensive Care Unit.

\subsubsection{Structure of Contaminant Species in Hospital}

During the study period, 20 pathogens were found to be responsible for BCC, these being (from most frequently isolated to the least): S. epidermidis, S. hominis, S. haemolyticus, S. capitis, Kocuria spp., S. warneri, S. auricularis, Micrococcus spp., Achromobacter xylosoxidans, Bacillus species, Granulicatella adiacens, Lactococcus garvieae, Pediococcus pentosaceus, Prevotella disiens, Propionibacterium acnes, S. caprae, S. cohnii, S. pseudintermedius, Streptococcus mitis, and Streptococcus salivarius. The most commonly isolated pathogen in BCC samples was Staphylococcus epidermidis (37.33\%), which together with three other coagulase-negative staphylococci (S. hominis, S. haemolyticus, and S. capitis) accounted for $84.3 \%$ of BCC (according to the rank selection) relative to all hospital-wide BCC (Figure 3).

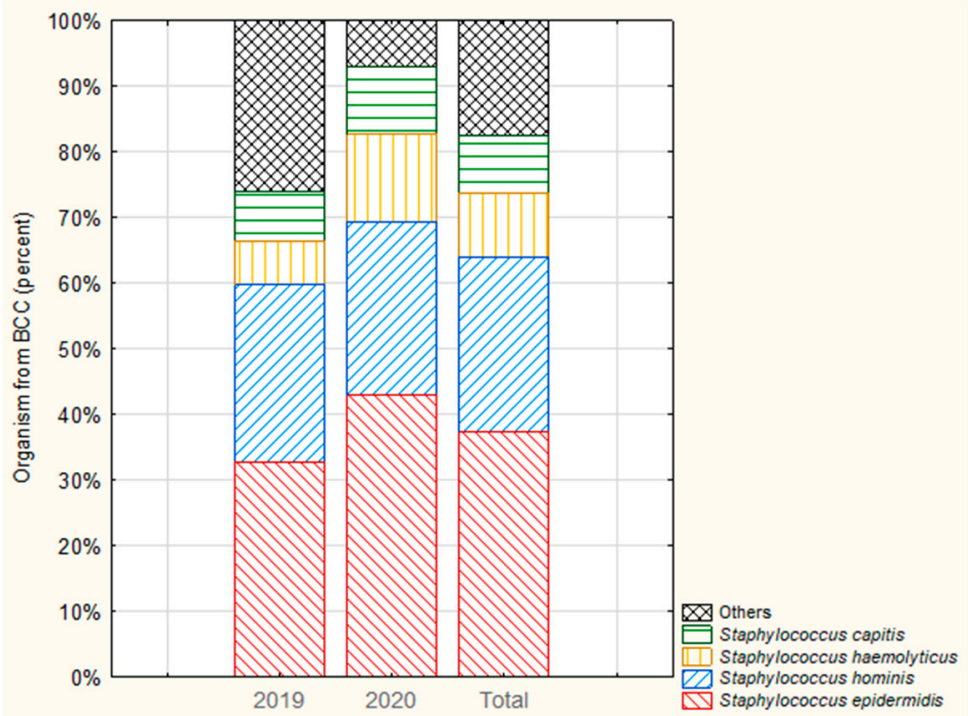

Figure 3. Distribution of the four most prevalent organisms isolated from blood culture contamination (BCC) in 2019 and 2020. 


\subsection{BCC vs. Critical Points for Obtaining a Clinically Insignificant BC Result}

The following analyses refer to the IM and ICU departments; these two most frequently collected $\mathrm{BC}$ for testing and were hence the main BCC contributors in the entire hospital. Two approaches were used to present the data: (1) for two departments combined ("IM + ICU") as a cumulative source of BCC in a hospital, and (2) data were collected separately for IM and ICU (2-year perspective or comparing 2019 to 2020).

\subsubsection{BCC by Month and Day of the Week}

Contamination rates for the two departments combined (IM and ICU) varied from month to month over the 2-year study period; however, no significant difference in BCC rate was found between months $(p>0.05)$. In 2019, the highest BCC rates were observed in January, February, and April (10.8\% in each) and the lowest in August and September ( $4.8 \%$ in both). In 2020, the highest BCC rate was recorded in December $(17.35 \%)$, while no contaminated samples were identified in April (Figure 4a).

The BCC rate for the total number of contaminations in the IM and ICU units according to weekday differed between 2019 and 2020. In 2019, the highest rate of BCC was recorded for Wednesday $(21.7 \%)$ and the lowest for Monday $(8.4 \%)$, while in 2020, the highest BCC was observed on Tuesday (25.4\%) and the lowest on Monday (8.5\%) (Figure $4 \mathrm{~b}$ ). However, no statistically significant difference in contamination rate was observed between different weekdays $(p>0.05)$.

Over the whole 2-year period, a higher BCC rate was observed in each of the departments during the working week (Monday-Friday), even when taking into account the different number of days per part of the week. However, this difference was only significant for the ICU $(p<0.05)$, with rates of $75.6 \%$ in the week and $24.4 \%$ in the weekend (Figure $5 \mathrm{a}$ ).

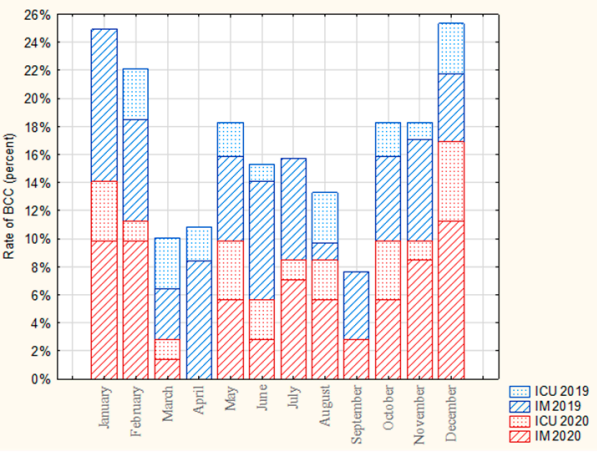

(a)

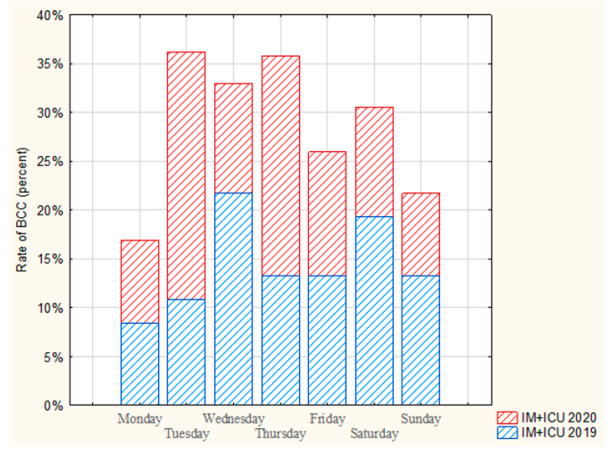

(b)

Figure 4. The rate of BCC for IM and ICU in 2019 and 2020 by: (a) month, (b) day of the week.

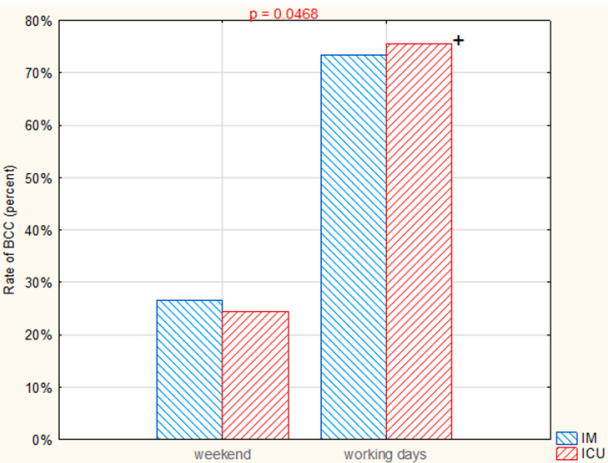

(a)

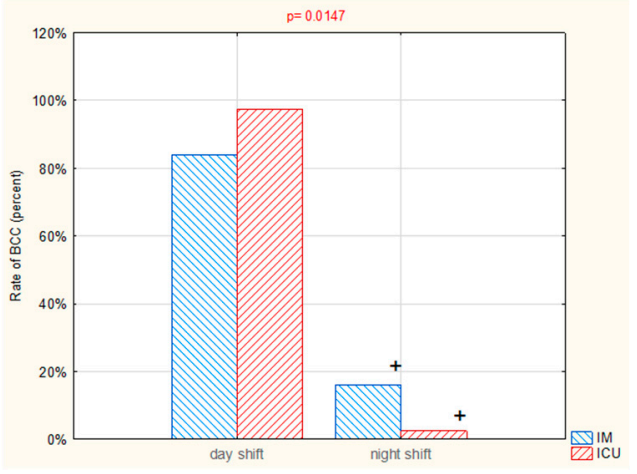

(b)

Figure 5. Rate of BCC on IM and ICU in 2019-2020 according to: (a) part of the week (working days vs. weekend), (b) type of shift (day vs. night). +: $p<0.05$. 


\subsubsection{BCC by Shift (Day vs. Night)}

The rate of BCC in both the IM and ICU differed between the night shift and the day shift during the 2-year period. As suspected, each department generated more BCCs on the day shift than on the night shift (IM: $82.3 \%$ vs. $17.7 \%$, ICU: $97.6 \%$ vs. $2.4 \%$, day vs. night respectively; $p<0.05$ ) (Figure $5 b$ ).

\subsubsection{Relation of Number of BC Sets to BCC Frequency}

In the IM department, in 2019, single sets (1/1) were used frequently (64\%) for routine diagnosis, and nearly $30 \%$ of these single sets demonstrated BCC. Even more single sets were used in $2020(84.7 \%)$, and the proportion of these sets with BCC was nearly 10\% higher than in the previous year (Figure 6) $(p<0.05)$.

In contrast, ICUs in 2019 mainly used double, or more than two $(1 / 2+)$, blood culture sets $(92.5 \%)$; additionally, no contamination was found in single sets, but at least double sets with BCC (1/2+) accounted for $50 \%$ of all sets. In 2020, BCC was recorded in nearly $13 \%$ of single $(1 / 1)$ sets; however, no significant, change was observed in the profile of BC sets used, compared with 2019 (Figure 6) $(p>0.05)$.

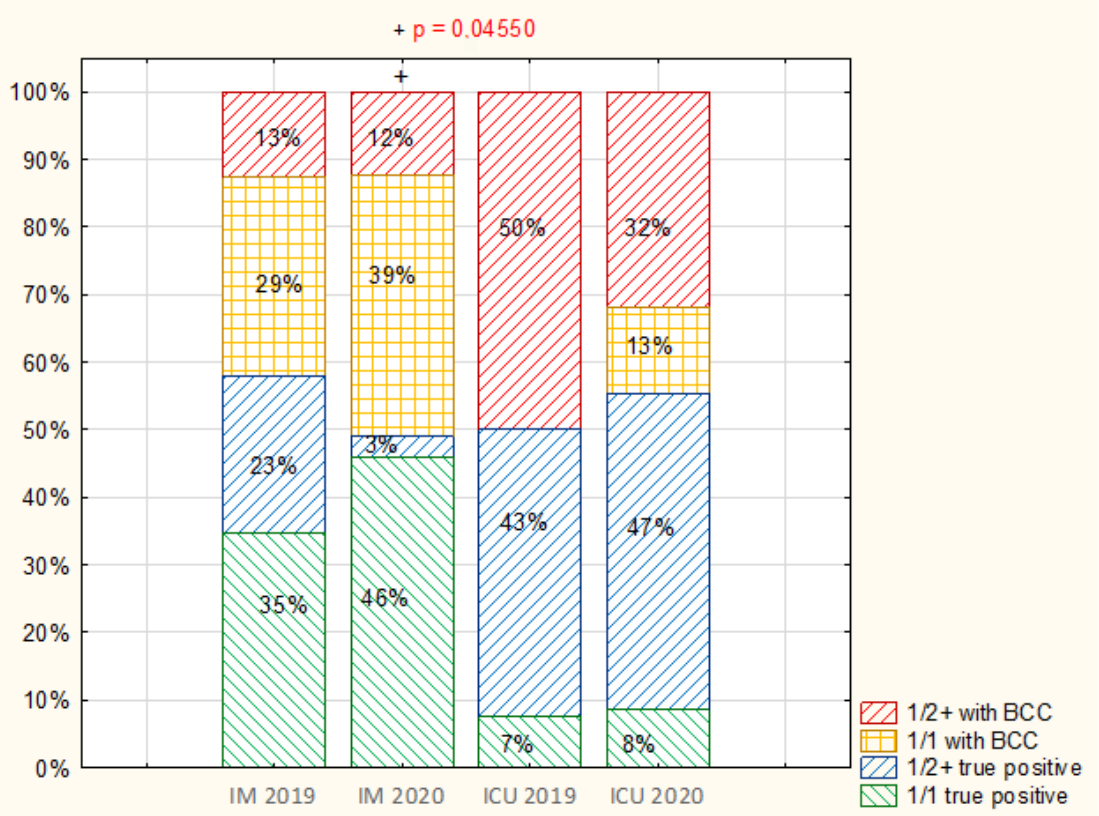

Figure 6. Comparison of the percentage of single (1/1) and the percentage of at least double $(1 / 2+)$ sets with BCC and with infection (true positive) (analysis without negative samples) in the Internal Medicine (IM) department and Intensive Care Unit (ICU) in 2019 and 2020.

\subsubsection{BCC by Organism}

In both departments, the most commonly isolated microorganisms from contamination in both 2019 and 2020 were coagulase-negative staphylococci. In 2019, the most prevalent species isolated from BCC on the IM department was S. epidermidis (39.1\%); in 2020, it was also S. epidermidis (42.4\%) (Figure 7a). In the ICU, S. haemolyticus was most commonly isolated in BCC samples: 37.5\% and 46.2\% (in 2019 and 2020 respectively) (Figure 7b). 


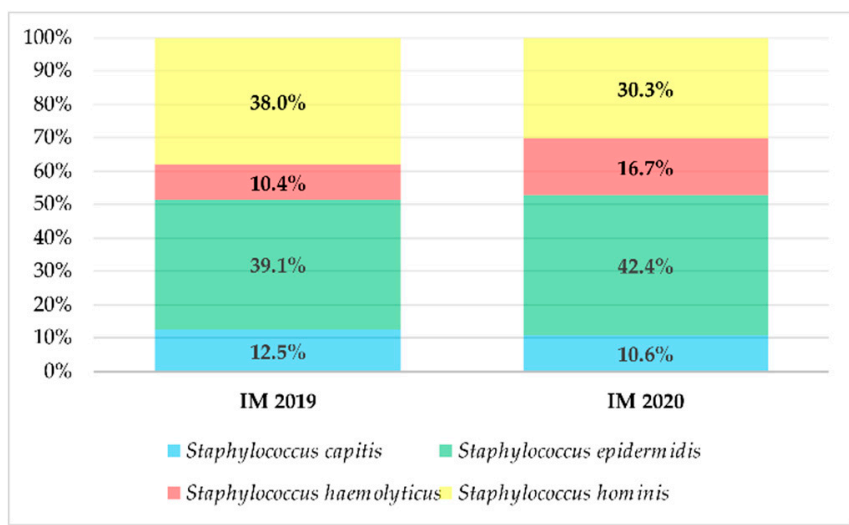

(a)

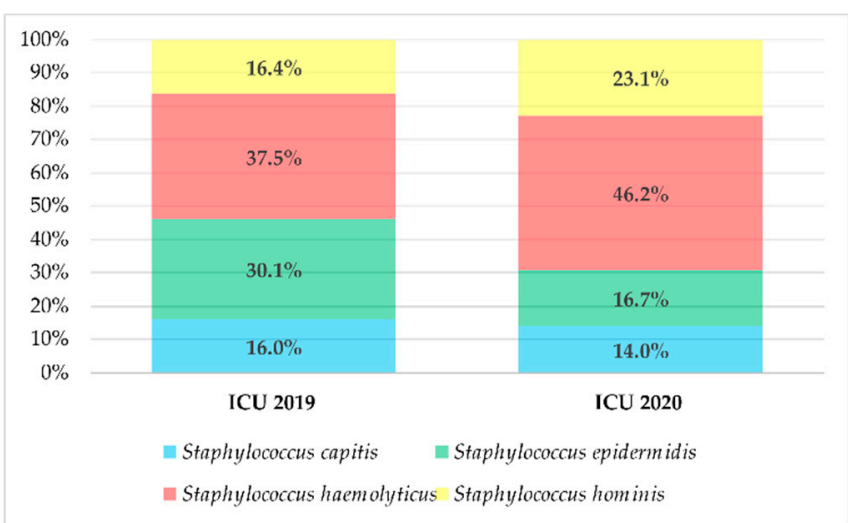

(b)

Figure 7. Distribution of the four major species of blood culture contaminants in 2019 and 2020 in the: (a) Internal Medicine (IM) department, (b) and Intensive Care Unit (ICU).

\subsection{Laboratory Costs Due to Contamination (Analysis for All Hospital Departments)}

The additional financial burden of contamination for the entire hospital was 61,229 PLN over 2 years. Taking into account the average euro exchange rate provided by the National Bank of Poland for 2019 and 2020, respectively, it was calculated that the total laboratory cost of BCC for the 2 years was EUR 268,036.40.

The highest costs were generated by the IM and ICU departments, together accounting for nearly $75 \%$ of the total laboratory costs related to BCC (i.e., 45353 PLN) (Table 3). The costs due to contamination increased from 28,427 PLN in 2019 to 32,802 PLN in 2020.

Table 3. Hospital laboratory cost profile due to occurrence of blood culture contamination in 2019 and 2020.

\begin{tabular}{cccccc}
\hline \multirow{2}{*}{ Department } & \multicolumn{2}{c}{ 2019 } & \multicolumn{2}{c}{ 2020 } & Total Cost of \\
& Real & Extra & Real & Extra & BCC [PLN] \\
\cline { 2 - 4 } $\begin{array}{c}\text { Internal } \\
\text { Medicine }\end{array}$ & 7021 & 5428 & 9702 & 7700 & 29,851 \\
\hline ICU & 4974 & 4214 & 3080 & 3234 & 15,502 \\
\hline Oncology & 1824 & 599 & 3080 & 924 & 6427 \\
\hline Infant Care & 783 & 522 & 693 & 462 & 2460 \\
\hline Neurology & 251 & 1091 & 308 & 1386 & 3036 \\
\hline Cardiology & 425 & 251 & 616 & 308 & 1600 \\
\hline $\begin{array}{c}\text { Infectious } \\
\text { Disease }\end{array}$ & 522 & 87 & 462 & 77 & 1148 \\
\hline Others & 174 & 261 & 308 & 462 & 1205 \\
\hline Total & 15,974 & 12,453 & 18,249 & 14,553 & 61,229 \\
\hline BCC-Blood Culture Contamination, ICU-Intensive Care Unit. & & \\
\hline
\end{tabular}

\section{Discussion}

Very few studies have attempted to assess the true scale of the problem of BCC, and none have made a simultaneous assessment of the possible financial consequences of sample contamination. Our present findings indicate that a high rate of BCC is not only a serious financial problem and a challenge for hospital managers, but also a potentially serious threat to the health and life of the patient. Identifying the source of BCC is very important in the diagnosis and management of patients with a suspicion of BSI. Controlling the frequency and sources of contamination, and taking steps to reduce it, can also minimize 
the unwarranted prescription of antibiotic therapy and the associated consequences of increasing drug resistance and adverse side effects. An analysis of BC results is a valuable source of information about the epidemiological status of a hospital [21,26]. It also makes it possible to assess compliance with microbiological sampling procedures.

Our findings indicate that the incidence of BCC for the entire hospital was $8.81 \%$ in 2019 and $10.62 \%$ in 2020. These results were much higher than the recommended target rate of $3 \%$ for $\mathrm{BCC}$ in adults [10]. Lalezari et al. report that $50 \%$ of all positive blood cultures were found to contain contaminants [27]. Washer et al. found $13 \%$ of all positive blood cultures appeared to be contaminated, and that overall blood contamination rates were $0.8 \%$ among cultures obtained peripherally by phlebotomists [19]. Rupp et al. found contamination in $23 \%$ of all positive blood cultures, and that overall contamination rates were $1.8 \%$ during a defined study period [11]. Story-Roller and Weinstein found contamination in $26 \%$ of all positive blood cultures and the overall contamination rate to be $3.9 \%$ [28].

It can be seen that the scope and magnitude of blood culture contamination is influenced by a range of factors. Efforts to reduce the rate of BCC should begin with identifying the departments that generate the most BC contamination. Targeting busy departments with high rates of BCC, such as IM and ICU, and implementing specific measures as a part of quality improvement interventions, will effectively reduce the rate of culture contamination [14]. The scale of this problem is also underscored by the fact that the rate of BCC observed in the study was close to true positive BC (11.70\% in 2019 and $10.62 \%$ in 2020).

The bacteria of the natural physiological microbiota of patients and staff can be transferred to transport media during venipuncture, and this contamination can be diagnosed as BCC. The most common bacteria isolated from BCC were coagulase-negative staphylococci (CoNS) (84.3\%), followed by other skin contaminants; this is in line with the results of similar studies $[8,9,13]$. For example, Weinstein et al. isolated a number of organisms representing contamination from adult patients in three hospitals around the USA: CoNS, Corynebacterium species, Bacillus species other than Bacillus anthracis, Propionibacterium acnes, Micrococcus species, viridans group streptococci, enterococci [29,30]. Our present data indicate that Staphylococcus epidermidis was the most frequent contaminant, which is consistent with the results in other papers $[8,14]$. According to the laboratory procedures, every contaminated $\mathrm{BC}$ result was consulted with a doctor, regardless of the number of blood cultures set.

A key aim of the study was to identify the key critical issues that may result in a higher incidence of BCC. Hence, the time of sampling was regarded as a critical point, more specifically month, day of the week and day/night shift. Regarding the variation across months during the 2-year follow-up period, no variation in BCC level was observed. This is in line with Hemeg et al., who also do not report any significant difference in contamination rates between months over a span of 1 year [8]. In contrast, Min et al. reported significant differences in contamination rates between months, with the highest rates seen in August and November and the lowest in February [31]. It can only be assumed that the number of samples from BCC will be higher in the summer months (vacation season), when there are staff shortages and the hospital is supported by external staff not permanently employed at the facility. However, no studies, if any, have examined whether the BCC rate is higher in any particular month during the year or on any days during the work week.

When the week was divided into workdays and weekends, only the ICU demonstrated a higher rate of BCC during workdays compared with weekends (more routine duties, pace of work). We suspected that the rate of BCC will be the highest on Monday due to the heavy workload after the weekend; however, no significant difference was observed. In addition, no statistically significant difference in contamination rate was found between different weekdays; this can be caused by the multitude of factors associated with the collection procedure, e.g., personal factors, lack of trained personnel, and employee rotation. The influence of the human factor (number of specialized personnel, age, seniority, checking knowledge of the procedures) should be taken into account in further studies. It can be 
assumed that a higher number of specialized personnel can contribute to a decrease in the number of incorrect BC collections.

Our study is one of the few to evaluate whether the BCC rate differs between day and night work shifts. The departments with the highest numbers of contaminations also demonstrated significantly more frequent clinically insignificant microbiological test results in blood during the day shift. This was likely related to the increased workload during the day shift, i.e., more patients to be cared for and more admissions to the department, as well as too few employees and employee rotations, which may have led to inadequate adherence to BC collection procedures. However, Jusoh et al. report a significantly higher risk of BCC during night shift work in the emergency department: BC collected during the night shift was almost five times more likely to be contaminated than that collected during the morning shift [32].

In the present study, our profile analysis found that the IM department used mainly single sets for collecting blood for cultures. As a result, the patients in this department were more likely to obtain a BCC result than those in the ICU department, and hence require another examination and a longer hospital stay, i.e., by 5 days on average $[9,13]$. This problem was most likely observed because of an insufficient understanding of the blood sampling procedures by staff on clinical departments. The time needed to repeat the test may play a role, as well as the necessity to perform other laboratory tests that may be helpful in diagnosing BSI, e.g., determination of C-reactive protein or procalcitonin levels. Any delay in making the correct diagnosis carries certain consequences [33]. Ferrer et al. found a delay in the administration of the first targeted antibiotic due to improperly collected specimens to be significantly related to an increase in in-hospital mortality in patients with severe sepsis and septic shock [6].

Since bacteria and fungi may not be constantly present in the bloodstream during infection, the sensitivity of a single blood culture set is very limited. In our study, 9.5\% of the single blood culture sets collected at the IM and ICU departments were true positive, while $44.83 \%$ of double sets were true positive. For samples with more than two sets, the percentage was $29 \%$ (data not shown in table). However, no attempt was made to determine the cumulative sensitivity of blood cultures in the present study. Studies performed in the 1970s, 1980s, and early 1990s found that performing two to three blood cultures from samples obtained from adults during a 24-h period could detect $99 \%$ of all BSIs [22,33-38]. Cockerill et al. reported that two blood cultures detected only $80 \%$ of BSIs, three detected $96 \%$ of BSIs, and four were required to detect $100 \%$ of BSIs; however, these results were unexpected given the use of a modern CMBCS (continuous-monitoring blood culture systems) and contemporary culture media. The authors suggest that newer systems may detect bacteremia at lower levels than older systems and that higher numbers of blood cultures should be used to detect low-level bacteremia [36]. Similar results were obtained by Lee at al., in an evaluation of the cumulative sensitivity of BC collected sequentially over $24 \mathrm{~h}$ using monitored automated systems; it was found that the cumulative yield of pathogens from three blood culture sets (two bottles per set), with a blood volume of $20 \mathrm{~mL}$ in each set (10 mL per bottle), was $73.2 \%$ for the first set, $93.9 \%$ for two sets, and $96.9 \%$ for three sets. To achieve a detection rate of $>99 \%$, as many as four blood cultures may be needed [22].

Unlike previous studies, our present paper also evaluates the additional laboratory costs caused by contamination in BC samples; however, this value is only a component part of the total additional cost associated with BCC. The overall structure of additional costs should take into account the costs of medications used, including antibiotics that were prescribed due to a clinically irrelevant result, the prolonged diagnostic process, and those associated with prolonged hospitalization, including costs related to waiting time for a repeat result of a microbiological blood test or other ancillary tests. Unfortunately, no detailed data on the amount of costs listed were available, and this constitutes a limitation to our present analysis. Gander et al. reported a difference in median patient charges between 
negative and false positive episodes of USD 8720 per contamination (a $47 \%$ increase); however, in this study, laboratory costs were not separated from the other expenses [18].

In contrast, Alnami et al. found a four-fold increase in microbiology charges per BCC compared with a negative BC [14]. In the latter case, the calculation takes into account the fact that an initial positive culture result does not yet indicate whether the result is true or false; therefore, each retest and subsequent establishment of the antimicrobial susceptibility profile involves further costs. These costs can be avoided for the negative result, provided it is not contaminated. Thus, even if the result ultimately turns out to be clinically insignificant (false positive, BCC), this does not remove the need to perform the next step of the test according to the procedure (real cost of BCC), as the first step could not rule out the possibility of a true positive result (infection).

The study has some limitations. Firstly, the number of BC samples was limited due to a change in the IT system in the hospital laboratory, resulting in a loss of data prior to 2019. In addition, many single sets of BC may be not fully informative. Finally, the study is retrospective in nature (stagnant data), thus yielding less data about the clinical status of the patient. However, the key strength of the study is its comprehensiveness, performing a simultaneous epidemiological, microbiological, and economic analysis associated with the occurrence of BCC.

\section{Conclusions}

The BCC rates for the hospital significantly exceeded internationally acceptable levels; the IM and ICU departments ordered the most collections, generating the highest number of contaminations. In order to reduce the rate of BCC and thus lower extra hospital costs, greater adherence to the BC collection procedure should be encouraged, e.g., correct disinfection of skin, and strictly adhering to the rule of using at least two BC sets. Although the study is limited by the use of single blood culture sets, the rate of BCC is very high. Our findings underline the need for greater staff training in this regard and much better communication between the laboratory and the hospital departments. Further prospective studies are needed to identify personal and organizational factors related to higher rates of BCC.

Author Contributions: Conceptualization, A.T. and A.G.-S.; methodology, A.T., R.D. and A.G.-S.; software, R.D.; validation, A.T., M.Ł. and A.G.-S.; formal analysis, R.D.; investigation, A.T., M.Ł., R.D. and A.G.-S.; resources, A.T. and A.G.-S.; data curation, A.T.; writing-original draft preparation, A.T.; writing-review and editing, A.T., M.Ł. and A.G.-S.; visualization, A.T., R.D. and A.G.-S.; supervision, M.Ł. and A.G.-S.; project administration, A.T.; funding acquisition, A.T. All authors have read and agreed to the published version of the manuscript.

Funding: This research was supported by project "InterDoktorMen-Building new quality and effectiveness of education in the formula of doctorate studies for healthcare managers at the Health Sciences Faculty at the Medical University in Łódź" (POWR.03.02.00-00-I027/16) and co-financed from European Union resources from the European Social Fund as a part of the Operational Program Knowledge Education Development.

Institutional Review Board Statement: Not applicable.

Informed Consent Statement: Not applicable.

Data Availability Statement: The data presented in this study are available on request from the corresponding author.

Acknowledgments: This research was conducted within the EU-financed InterDoktorMen project.

Conflicts of Interest: The authors declare no conflict of interest. 


\section{References}

1. Ziegler, M.J.; Pellegrini, D.C.; Safdar, N. Attributable mortality of central line associated bloodstream infection: Systematic review and meta-analysis. Infection 2015, 43, 29-36. [CrossRef] [PubMed]

2. Heczko, P.; Wróblewska, M.; Pietrzyk, A. Zakażenia układowe i narządowe. In Mirobiologia Lekarska; Wydawnictwo Lekarskie PZWL: Warszawa, Poland, 2014; Volume 1, pp. 743-754.

3. Vincent, J.L.; Sakr, Y.; Sprung, C.L.; Ranieri, V.M.; Reinhart, K.; Gerlach, H.; Moreno, R.; Carlet, J.; Le Gall, J.R.; Payen, D. Sepsis Occurrence in Acutely Ill Patients Investigators. Sepsis in European intensive care units: Results of the SOAP study. Crit. Care Med. 2006, 34, 344-353. [CrossRef] [PubMed]

4. Martinez, R.M.; Wolk, D.M. Bloodstream Infections. Microbiol. Spectr. 2016, 4, 4. [CrossRef] [PubMed]

5. Khatib, R.; Saeed, S.; Sharma, M.; Riederer, K.; Fakih, M.G.; Johnson, L.B. Impact of initial antibiotic choice and delayed appropriate treatment on the outcome of Staphylococcus aureus bacteremia. Eur. J. Clin. Microbial. Infect. Dis. 2006, 25, 81-85. [CrossRef]

6. $\quad$ Ferrer, R.; Martin-Loeches, I.; Phillips, G.; Osborn, T.M.; Townsend, S.; Dellinger, R.P.; Artigas, A.; Schorr, C.; Levy, M.M. Empiric antibiotic treatment reduces mortality in severe sepsis and septic shock from the first hour: Results from a guideline-based performance improvement program. Crit. Care Med. 2014, 42, 1749-1755. [CrossRef]

7. El Feghaly, R.E.; Chatterjee, J.; Dowdy, K.; Stempak, L.M.; Morgan, S.; Needham, W.; Prystupa, K.; Kennedy, M. A Quality Improvement Initiative: Reducing Blood Culture Contamination in a Children's Hospital. Pediatrics 2018, 142, e20180244. [CrossRef]

8. Hemeg, H.A.; Almutairi, A.Z.; Alharbi, N.L.; Alenezi, R.F.; Alturkostani, M.A.; Ozbak, H.A.; Islam, F.A. Blood culture contamination in a tertiary care hospital of Saudi Arabia. A one-year study. Saudi Med. J. 2020, 41, 508-515. [CrossRef]

9. Doern, G.V.; Carroll, K.C.; Diekema, D.J.; Garey, K.W.; Rupp, M.E.; Weinstein, M.P.; Sexton, D.J. Practical Guidance for Clinical Microbiology Laboratories: A Comprehensive Update on the Problem of Blood Culture Contamination and a Discussion of Methods for Addressing the Problem. Clin. Microbiol. Rev. 2019, 33, e00009-19. [CrossRef]

10. Wayne, P.A. Principles and Procedures for Blood Cultures. Approved Guideline, CLSI Document M47-A. Clinical and Laboratory Standards Institute (CLSI), 2007. Available online: https:/ / clsi.org/media/1448/m47a_sample.pdf (accessed on 30 May 2021).

11. Rupp, M.E.; Cavalieri, R.J.; Marolf, C.; Lyden, E. Reduction in Blood Culture Contamination Through Use of Initial Specimen Diversion Device. Clin. Infect. Dis. 2017, 65, 201-205. [CrossRef]

12. Hall, K.K.; Lyman, J.A. Updated review of blood culture contamination. Clin. Microbiol. Rev. 2006, 19, 788-802. [CrossRef]

13. Alahmadi, Y.M.; Aldeyab, M.A.; McElnay, J.C.; Scott, M.G.; Darwish Elhajji, F.W.; Magee, F.A.; Dowds, M.; Edwards, C.; Fullerton, L.; Tate, A.; et al. Clinical and economic impact of contaminated blood cultures within the hospital setting. J. Hosp. Infect. 2011, 77, 233-236. [CrossRef] [PubMed]

14. Alnami, A.Y.; Aljasser, A.A.; Almousa, R.M.; Torchyan, A.A.; BinSaeed, A.A.; Al-Hazmi, A.M.; Somily, A.M. Rate of blood culture contamination in a teaching hospital: A single center study. J. Taibah Univ. Med. Sci. 2015, 10, 432-436. [CrossRef]

15. Self, W.H.; Talbot, T.R.; Paul, B.R.; Collins, S.P.; Ward, M.J. Cost analysis of strategies to reduce blood culture contamination in the emergency department: Sterile collection kits and phlebotomy teams. Infect. Control Hosp. Epidemiol. 2014, 35, 1021-1028. [CrossRef]

16. Garcia, R.A.; Spitzer, E.D.; Beaudry, J.; Beck, C.; Diblasi, R.; Blabac, M.; Haugaard, C.; Heuschneider, S.; Kranz, B.P.; McLean, K.; et al. Multidisciplinary team review of best practices for collection and handling of blood cultures to determine effective interventions for increasing the yield of true-positive bacteremias, reducing contamination, and eliminating falsepositive central line-associated bloodstream infections. Am. J. Infect. Control 2015, 43, 1222-1237. [PubMed]

17. Dargère, S.; Cormier, H.; Verdon, R. Contaminants in blood cultures: Importance, implications, interpretation and prevention. Clin. Microbiol. Infect. 2018, 24, 964-969. [CrossRef]

18. Gander, R.M.; Byrd, L.; DeCrescenzo, M.; Hirany, S.; Bowen, M.; Baughman, J. Impact of blood cultures drawn by phlebotomy on contamination rates and health care costs in a hospital emergency department. J. Clin. Microbiol. 2009, 47, 1021-1024. [CrossRef]

19. Washer, L.L.; Chenoweth, C.; Kim, H.W.; Rogers, M.A.; Malani, A.N.; Riddell, J.; Kuhn, L., 4th; Noeyack, B.; Neusius, H., Jr.; Newton, D.W.; et al. Blood culture contamination: A randomized trial evaluating the comparative effectiveness of 3 skin antiseptic interventions. Infect. Control Hosp. Epidemiol. 2013, 34, 15-21. [CrossRef]

20. Pien, B.C.; Sundaram, P.; Raoof, N.; Costa, S.F.; Mirrett, S.; Woods, C.W.; Reller, L.B.; Weinstein, M.P. Evaluation of the microbiology, epidemiology, and outcome of bloodstream infections in hospitalized adults. Am. J. Med. 2010, 123, 819-828. [CrossRef]

21. Richter, S.S.; Beekman, S.E.; Croco, D.J.; Koontz, R.P.; Pfaller, M.A.; Doern, G.V. Minimizing the workup of blood culture contaminants: Implementation and evaluation of a laboratory-based algorithm. J. Clin. Microbiol. 2002, 40, 2437-2444. [CrossRef]

22. Lee, A.; Mirrett, S.; Reller, L.B.; Weinstein, M.P. Detection of bloodstream infections in adults: How many blood cultures are needed? J. Clin. Microbiol. 2007, 45, 3546-3548. [CrossRef]

23. Self, W.H.; Speroff, T.; Grijalva, C.G.; McNaughton, C.D.; Ashburn, J.; Liu, D.; Arbogast, P.G.; Russ, S.; Storrow, A.B.; Talbot, T.R. Reducing blood culture contamination in the emergency department: An interrupted time series quality improvement study. Acad. Emerg. Med. 2013, 20, 89-97. [CrossRef] [PubMed]

24. Bartoszewicz, M. Zasady diagnostyki zakażeń krwi. In Rekomendacje Grupy Ekspertów pod Redakcją Marzenny Bartoszewicz; Evereth Publishing: Warszawa, Poland, 2020; Volume 1, pp. 14, 21-24. 
25. The European Committee on Antimicrobial Susceptibility Testing_EUCAST. Available online: https://www.eucast.org/ast_of_ bacteria/previous_versions_of_documents / (accessed on 30 December 2021).

26. Wałaszek, M.; Różańska, A.; Bulanda, M.; Wojkowska-Mach, J. Polish Society of Hospital Infections Team. Alarming results of nosocomial bloodstream infections surveillance in Polish intensive care units. Przegl. Epidemiol. 2018, 72, 33-44.

27. Lalezari, A.; Cohen, M.J.; Svinik, O.; Tel-Zur, O.; Sinvani, S.; Al-Dayem, Y.A.; Block, C.; Moses, A.E.; Oster, Y.; Salameh, S.; et al. A simplified blood culture sampling protocol for reducing contamination and costs: A randomized controlled trial. Clin. Microbiol. Infect. 2020, 26, 470-474. [CrossRef] [PubMed]

28. Story-Roller, E.; Weinstein, M.P. Chlorhexidine versus tincture of iodine for reduction of blood culture contamination rates: A prospective randomized crossover study. J. Clin. Microbiol. 2016, 54, 3007-3009. [CrossRef]

29. Weinstein, M.P. Blood culture contamination: Persisting problems and partial progress. J. Clin. Microbiol. 2003, 41, 2275-2278. [CrossRef] [PubMed]

30. Weinstein, M.P.; Towns, M.L.; Quartey, S.M.; Mirrett, S.; Reimer, L.G.; Parmigiani, G.; Reller, L.B. The clinical significance of positive blood cultures in the 1990s: A prospective comprehensive evaluation of the microbiology, epidemiology, and outcome of bacteremia and fungemia in adults. Clin. Infect. Dis. 1997, 24, 584-602. [CrossRef] [PubMed]

31. Min, H.; Park, C.S.; Kim, D.S.; Kim, K.H. Blood culture contamination in hospitalized pediatric patients: A single institution experience. Korean J. Pediatr. 2014, 57, 178-185. [CrossRef]

32. Jusoh, A.F.; Zahary, M.N.; Yahaya, R.; Him, N.A.S.N.; Mohamed, N.A.N.; Hassan, S.A.; Fauzi, M.H.; Sheng, C.K. Blood culture contamination in emergency department: Association with working shift, triage and stroke. Rawal Med. J. 2018, 43, 23-28.

33. Chandrasekar, P.H.; Brown, W.J. Clinical issues of blood cultures. Arch. Intern. Med. 1994, 154, 841-849. [CrossRef]

34. Washington, J.A. Blood cultures: Principles and techniques. Mayo Clin. Proc. 1975, 50, 91-98.

35. Weinstein, M.P.; Reller, L.B.; Murphy, J.R.; Lichtenstein, K.A. The clinical significance of positive blood cultures: A comprehensive analysis of 500 episodes of bacteremia and fungemia in adults. I. Laboratory and epidemiologic observations. Rev. Infect. Dis. 1983, 5, 35-53. [CrossRef]

36. Cockerill, F.R.; Wilson, J.W., III; Vetter, E.A.; Goodman, K.M.; Torgerson, C.A.; Harmsen, W.S.; Schleck, C.D.; Ilstrup, D.M.; Washington, J.A., II; Wilson, W.R. Optimal testing parameters for blood cultures. Clin. Infect. Dis. 2004, 38, 1724-1730. [CrossRef] [PubMed]

37. Murray, P.R.; Masur, H. Current approaches to the diagnosis of bacterial and fungal bloodstream infections in the intensive care unit. Crit. Care Med. 2012, 40, 3277-3282. [CrossRef] [PubMed]

38. Souvenir, D.; Anderson, D.E.; Palpant, S.; Mroch, H.; Askin, S.; Anderson, J.; Claridge, J.; Eiland, J.; Malone, C.; Garrison, M.W.; et al. Blood cultures positive for coagulase-negative staphylococci: Antisepsis, pseudobacteremia, and therapy of patients. J. Clin. Microbiol. 1998, 36, 1923-1926. [CrossRef] [PubMed] 